\title{
Long-Term Femtosecond Timing Link Stabilization Using a Single-Crystal Balanced Cross-Correlator
}

\author{
Jungwon Kim ${ }^{1}$, Florian Löhl', Jeff Chen ${ }^{1}$, Zhigang Zhang ${ }^{1}$, Holger Schlarb ${ }^{2}$, Franco Wong ${ }^{1}$ and Franz Kärtner ${ }^{1}$ \\ ${ }^{1}$ Department of Electrical Engineering and Computer Science and Research Laboratory of Electronics \\ Massachusetts Institute of Technology, 77 Massachusetts Avenue, Cambridge, Massachusetts 02139 \\ Email: jungwon@mit.edu \\ ${ }^{2}$ Deutsches Elektronen-Synchrotron (DESY), Notkestrasse 85, 22607 Hamburg, Germany
}

\begin{abstract}
We demonstrate a self-aligned balanced cross-correlator based on a single type-II phase-matched PPKTP crystal and its use in timing link stabilization. We obtained long-term 10-fs timing stabilization of a 300-meter fiber link.

(C)2007 Optical Society of America

OCIS codes: (120.3940) Metrology; (320.7160) Ultrafast technology
\end{abstract}

Precise optical timing distribution to remote locations is important for large-scale facilities requiring high-precision synchronization, for example, seeded x-ray free electron lasers [1] and phased-array antennas [2]. It is also crucial for the optical time/frequency standard distribution over long distances [3]. Acoustic, thermal noise and drifts introduced by the fiber involved in the distribution must be removed. So far, electronic techniques based on highspeed photodetectors and microwave mixers are used to stabilize fiber links on a short-term time scale [4,5]. The limited timing resolution as well as strong thermal drifts of microwave components makes it difficult to stabilize the link in a long-term stable way.

In this work, a single type-II phase-matched PPKTP crystal is used to construct a compact and self-aligned balanced optical cross-correlator for precise timing detection. We used the timing error signal generated from this balanced cross-correlator, which is robust against intensity noise and temperature drifts, to stabilize a 300-meter fiber link. In a first experimental demonstration, $10 \mathrm{fs}$ level stabilization is achieved.

Previously, a balanced cross-correlator for long-term sub-fs synchronization of two independently mode-locked lasers was demonstrated [6]. However, this method is limited to the case of optical pulses with different center wavelengths. The self-aligned balanced cross-correlator in this work consists of (a) generation of a group delay difference between two orthogonally polarized pulses and (b) broadband second-harmonic generation (SHG) by a type-II phase-matched nonlinear crystal. Using the group delay from the birefringence between the two orthogonal polarizations in the crystal enables the implementation of balanced cross-correlation at the same wavelength. The detected signal is background free, i.e., if the pulses do not overlap in time, the detector signal is vanishing. Moreover, the group delay and SHG functions can be combined in a single nonlinear crystal. For construction of a cross-correlator at $1550 \mathrm{~nm}$, the use of a PPKTP crystal is especially advantageous because of the extended phasematching bandwidth of $100 \mathrm{~nm}$ centered at $1550 \mathrm{~nm}$ [7].
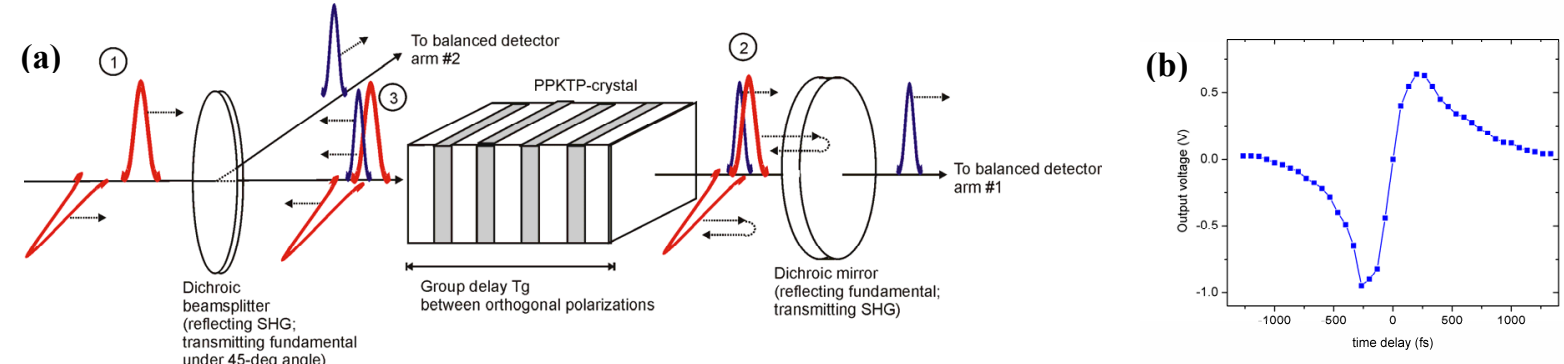

Figure 1: (a) Operation of a single-crystal balanced cross-correlator. (b) Autocorrelation trace using $0.34 \mathrm{~nJ}, 170-\mathrm{fs}$ pulses at $1550 \mathrm{~nm}$.

Figure 1 (a) shows the operation of a single-crystal balanced cross-correlator. The input pulses are transmitted through the first dichroic beamsplitter which transmits the input pulses but reflects the SHG of the input pulses. The pulses are focused into a type-II phase-matched PPKTP crystal. The generated SHG component is transmitted through the second dichroic mirror and detected by the photodiode 1 in the balanced detector. The remaining fundamental input pulses are reflected back by the dichroic mirror and again focused into the PPKTP crystal. The SHG component generated by the back-reflected pulses is separated by the 45-degree dichroic beamsplitter and detected by the photodiode 2 in the balanced detector. At the balanced detector output, a signal proportional to the relative position between the two input pulses is extracted. Figure 1 (b) shows the autocorrelation of a $0.34 \mathrm{~nJ}, 170$ fs pulse at $1550 \mathrm{~nm}$ using a 5-mm long PPKTP crystal with a poling period of $46.2 \mu \mathrm{m}$. 


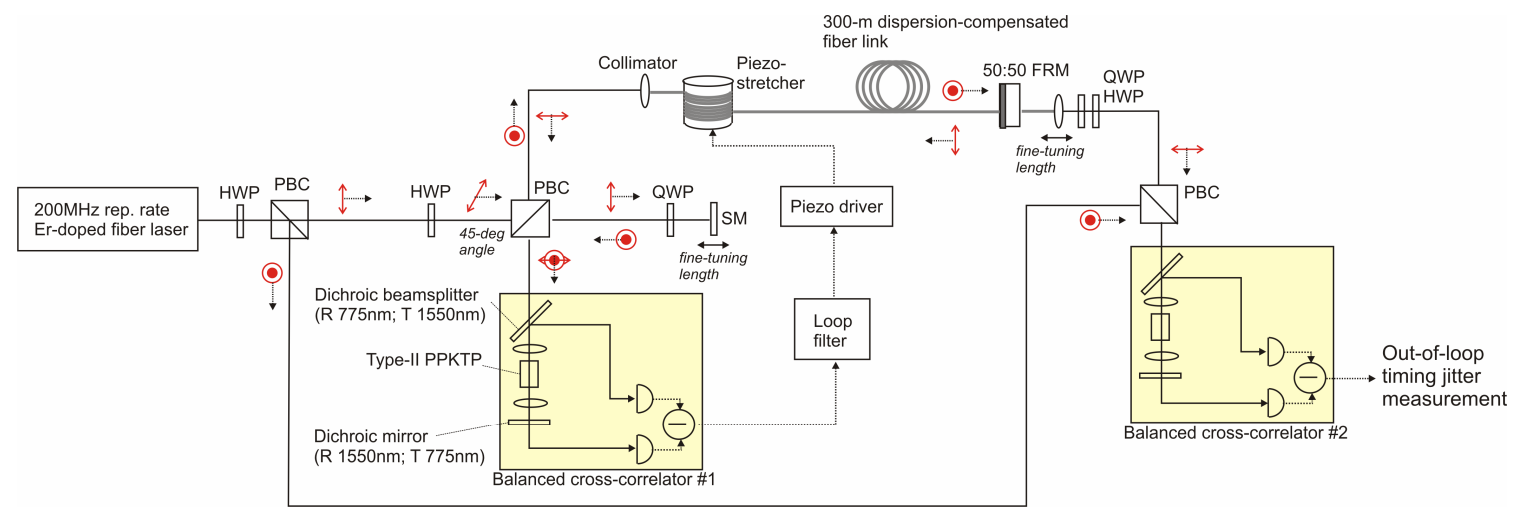

Figure 2: Schematic setup for timing link stabilization using a single-crystal balanced cross-correlator. FRM: Faraday rotating mirror, HWP: half-wave plate, PBC: polarization beam cube, QWP: quarter-wave plate, R: reflection, SM: silver mirror, T: transmission.

Figure 2 shows the schematic setup for a 300-meter timing link stabilization using the single-crystal balanced cross-correlator. A 200-MHz soliton Er-doped fiber laser is used as the optical pulse source. The output power is 40 $\mathrm{mW}$ and the pulsewidth is about $200 \mathrm{fs}$. Part of the input pulse train is transmitted through a 300-meter long dispersion-compensated fiber link with a piezo-stretcher. Half of the transmitted pulses are back-reflected by a 50:50 Faraday rotating mirror (FRM) at the end of the fiber link. With FRM, the polarization state of the returning pulse is orthogonal to that of the input pulse. The reflected pulse (measured pulsewidth $\sim 420 \mathrm{fs}$ ) is combined with the fresh pulse directly from the laser at the polarization beam cube. The combined pulses are applied to the balanced crosscorrelator. The error signal generated from the balanced cross-correlator is regulated by a loop filter and applied to the piezo-stretcher in the link via a high-voltage piezo driver. This closes the timing stabilization loop. To evaluate out-of-loop performances, a second balanced correlator is used to compare the transmitted pulses with fresh pulses.

(a)

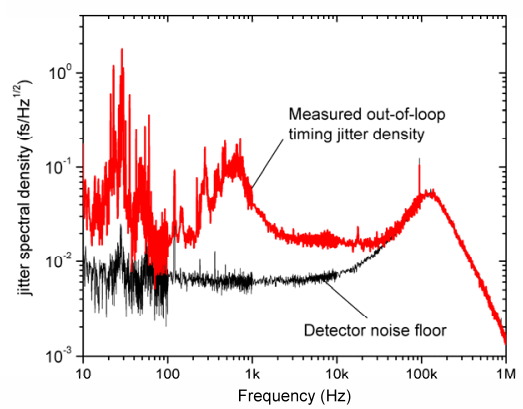

(b)

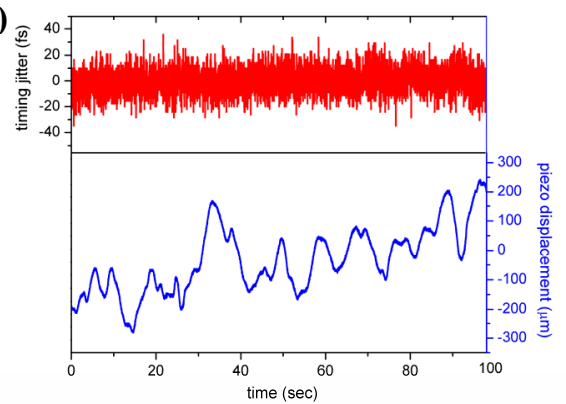

Figure 3: (a) Measured out-of-loop timing jitter density. (b) Long-term out-of-loop timing jitter trace over 100 seconds.

Figure 3 shows the measurement result of the stabilized fiber link. The out-of-loop rms-timing jitter integrated from $10 \mathrm{~Hz}$ to $100 \mathrm{kHz}$ (detector bandwidth) is $9.2 \mathrm{fs}$, where the detector background noise corresponds to $8.2 \mathrm{fs}$. The long-term out-of-loop timing jitter measured over 100 seconds showed $9.7 \mathrm{fs}$ level stabilization. Note that the fiber link is not temperature, vibration, nor airflow stabilized, and the locking is broken purely by the limited displacement range of the piezo stretcher we used $\left(\sim 1 \mathrm{~mm}\right.$ travel range corresponds to $3 \times 10^{-6}$ length fluctuation of the whole fiber link). With a proper manual adjustment of the translation stage, we could keep the lock for more than one hour. To our best knowledge, this result is the first long-term 10-fs level stabilization of a fiber link. It also indicates that adding a motorized translation stage and polarization control at the end of the fiber will enable to maintain this level of accuracy in synchronization over hours of operation.

In summary, we have demonstrated a self-aligned, single-crystal balanced cross-correlator comparing the timing between optical pulses at the same wavelength range and its use for long-term 10-fs precision stabilization of the 300 -meter fiber link. Further work to improve the long-term stability over several hours is in progress.

[1] J. Kim et al., Proceedings of FEL Conference 2004, p.329, August 2004.

[2] B. Shillue, Proceedings of IEEE/LEOS Summer Topical Meetings 2005, p. 61, July 2005.

[3] J. Ye et al., J. Opt. Soc. Am. B 20, 1459 (2003); K. W. Holman, D. J. Jones, D. D. Hudson and J. Ye, Opt. Lett. 29, 1554 (2004); K. W. Holman, D. D. Hudson, J. Ye and D. J. Jones, Opt. Lett. 30, 1225 (2005).

[4] A. Winter et al., Proceedings of FEL Conference 2005, p. 676, August 2005.

[5] D. D. Hudson, S. M. Foreman, S. T. Cundiff and J. Ye, Opt. Lett. 31, 1951 (2006).

[6] T. R. Schibli et al., Opt. Lett. 28, 947 (2003); J. Kim et al., Proceedings of EPAC 2006, p. 2744, June 2006.

[7] F. König and F. N. C. Wong, Appl. Phys. Lett. 84, 1644 (2004). 